\title{
DISCUSSION
}

\section{mouvements de terrains en Tarentaise}

\author{
par P. HABIB \\ Laboratoire de Mécanique des Solides \\ (EP - ENSMP - ENPC - CNRS) *
}

Rev. Franç. Géotech. n 53 , pp. 71.74 (octobre 1990)

Les remarques et observations présentées ici font référence à l'article de B. GOGUEL (RFG n 48 - pp. 55/64) sur les mouvements de terrains qui affectent le $\mathrm{Mt}$ Friolin depuis plusieurs années, mais il s'agit davantage d'un commentaire et d'un complément plutôt que d'une véritable discussion d'un phénomène dont la réalité paraît... indiscutable. Il est en effet frappant de constater, lorsqu'on observe le site, que l'interprétation des mouvements du Mt Friolin ne soulève aucune objection et l'on peut même dire que si la démonstration par Jean et Bernard GOGUEL d'un glissement en bloc a pu prendre plusieurs années, il suffit actuellement de regarder la pente naturelle avec cette idée en tête pour être immédiatement convaincu de l'existence du mouvement, même si ses causes géologiques et géotechniques restent peut-être encore à approfondir.

Mais on peut se demander si ce genre d'accident n'est pas beaucoup plus général qu'on ne l'imagine et si les régions montagneuses en général, et les Alpes en particulier, ne sont pas des domaines privilégiés pour la recherche de cicatrices en surface d'événements anciens ou même de mouvements actuels plus ou moins actifs, plus ou moins lents. Avec une collision Afrique-Europe à la vitesse d'au moins $1,5 \mathrm{~cm} /$ an les Alpes atteignent des hauteurs de $4000 \mathrm{~m}$, de même que l'Himalaya atteint $8000 \mathrm{~m}$ sous l'effet de la collision Inde-Asie à la vitesse d'au moins $3 \mathrm{~cm} / \mathrm{an}$. Il est probable que la surrection des montagnes se fait à des vitesses, plus petites, mais voisines, peut-être pour les Alpes de l'ordre de quelques $\mathrm{mm} / \mathrm{an}$.

L'usure des montagnes (c'est-à-dire la diminution de hauteur des montagnes) par érosion, glissement, effondrement... doit être aussi en moyenne du même ordre de grandeur. Mais si l'on peut - peut-être parler de vitesse moyenne pour le mouvement des plaques il est bien clair que les phénomènes de rééquilibrage des altitudes par des glissements de terrains sont essentiellement discontinus avec des périodes de crises séparées par des périodes de repos. Les Alpes doivent donc être couvertes de cicatrices de glissements anciens et l'on doit aussi y trouver de très nombreux mouvements actuels plus ou moins actifs.

- Ecole Polytechnique, 91128 Palaiseau Cedex.
A ce titre la vallée de l'Isère est particulièrement intéressante pour de telles recherches, car comme me l'a fait remarquer Jean PERA, Directeur du CETU, que je remercie ici pour les éléments bibliographiques qu'il m'a transmis et qui sont les seuls que j'ai pu trouver, le domaine du houiller en Tarentaise est éminemment sensible probablement parce que la résistance mécanique de ces formations est moindre que pour des terrains plus cristallisés ou plus cimentés. Il est par exemple extrêmement instructif de s'installer sur un belvédère en montagne par une journée ensoleillée avec une carte topographique et des jumelles et de regarder les pentes aux différentes heures de la journée de façon à pouvoir profiter de l'orientation de la lumière, depuis le soleil rasant du matin jusqu'à celui du soir, et de voir apparaitre puis se cacher les reliefs significatifs des mouvements de pentes. Ensuite, il n'y a plus qu'à aller voir sur le terrain pour apprécier si le mouvement est actif ou au contraire arrêté.

Dans le voisinage de Peisey Nancroix, c'est-à-dire très près du Mt Friolin, sur la rive droite de l'Isère, en face de la vallée de Peisey j'ai pu ainsi identifier et examiner plusieurs très grands glissements dont lun, situé au-dessus de Valezan est d'une ampleur exceptionnelle. Il a d'ailleurs déjà fait l'objet d'observations. Ainsi en 1872 le Baron Achille RAVERAT écrivait dans « La Savoie * (p. 428) :

«Entre Aime et Bellentre s'élève un coteau qui offre un phénomène digne de fixer l'attention du géologue et d'éveiller la sollicitude administrative. Le Neiget, dit M. Félix DESPINE, sous-préfet de Moûtiers, est un mas assez vaste, glissant peu à peu vers l'Isère en un seul bloc et entraînant par un mouvement lent, uniforme et continu, sans presque en déranger la symétrie, arbres, rochers, champs et maisons. Le même phénomène se montre sur plusieurs points de la Tarentaise. Ainsi non loin de Moûtiers, un mouvement analogue entraine les terrains sur lesquels repose Notre-Dame-du-Pré ; et l'église qui se voyait à peine depuis la plaine, il y a vingt cinq ans apparaît toute entière aujourd'hui ».

«Mais ce pays est charmant, une luxuriante verdure, une fertile végétation cachent aux yeux du voyageur un danger dont la population elle-même ne semble pas se préoccuper ; puis quelque jour, à la suite d'un 
terrible cataclysme on apprendra que plusieurs villages ont disparu de la surface du sol ».

Sous un langage fleuri on retrouve ainsi des considérations qui sont malheureusement toujours actuelles dans toutes les Alpes.

Le glissement de Valezan a été probablement identifié par J. FABRE et cité dans une note infra paginale de sa thèse "Contribution à l'étude de la zone houillère en Maurienne et en Tarentaise " (Editions
Technip - 1941, p. 16). De toute façon, il a été cartographié, en ce sens que les accidents de surface engendrés par une ou plusieurs coquilles de glissement figurent sur les cartes topographiques du site en rive droite de l'Isère ; la figure 1 indique les éléments cartographiques principaux, les accidents de surface topographiés, l'extension probable du glissement et une coupe dans l'axe moyen de l'accident. Le glissement s'étend juste en dessous d'un altiport (qui figure sur la carte Michelin) situé à $2225 \mathrm{~m}$ d'altitude

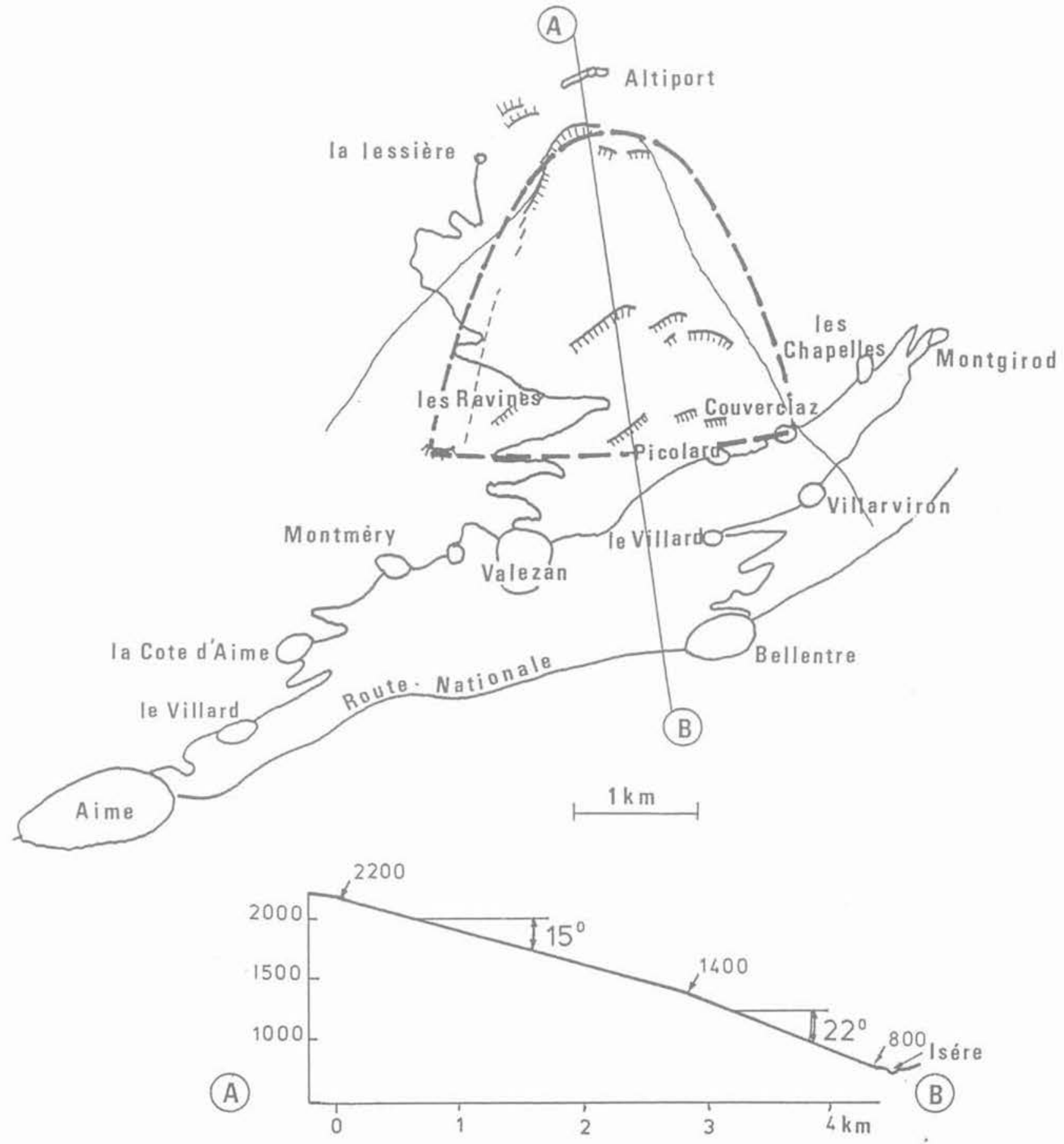

Fig. 1. - Plan général du site et de la zone en mouvement et profil du sol dans l'axe du glissement. 
jusqu'à la cote $1400 \mathrm{~m}$ au moins. Latéralement des points fixes ou des points durs sont visibles et il ne me semble pas que les exploitations d'anthracite exploitées aux Chapelles (Mines de Mongirod) situées à gauche du glissement, ni a fortiori les affleurements de charbon d'Aime et du Villard situés très à droite du glissement, c'est-à-dire à l'aval selon le cours de IIsère, aient le moindre rapport avec ce mouvement de terrain. Il est curieux que le talus qui a certainement glissé, et qui à mon avis continue à glisser, correspond à la pente la plus faible du coteau. La coupe de la figure 1 montre en effet que la pente moyenne entre 2200 et $1400 \mathrm{~m}$ est de $15^{\circ}$ alors que de $1400 \mathrm{~m}$ jusqu'à la route nationale à $800 \mathrm{~m}$ d'altitude, elle est de $22^{\circ}$. On remarque que la pente de $15^{\circ}$ est inférieure à celle des formations du houiller profond, c'est-à-dire aux grandes surfaces structurales locales, qui est plutôt de $25^{\circ}$ d'après J. FABRE. Les figures $2,3,4$ et 5 représentent l'allure générale du site ainsi que des vues particulières prises au cours de l'été 1989 qui a été dans les Alpes françaises, il faut le rappeler, une année d'enneigement tardif et très faible, ce qui a entraîné l'été suivant après la fonte des neiges un ralentissement systématique de tous les mouvements de terrains. On distingue cependant, sur le terrain, un certain nombre de marqueurs qui indiquent que ce glissement est toujours actif même si les vitesses de déplacement sont probablement petites.
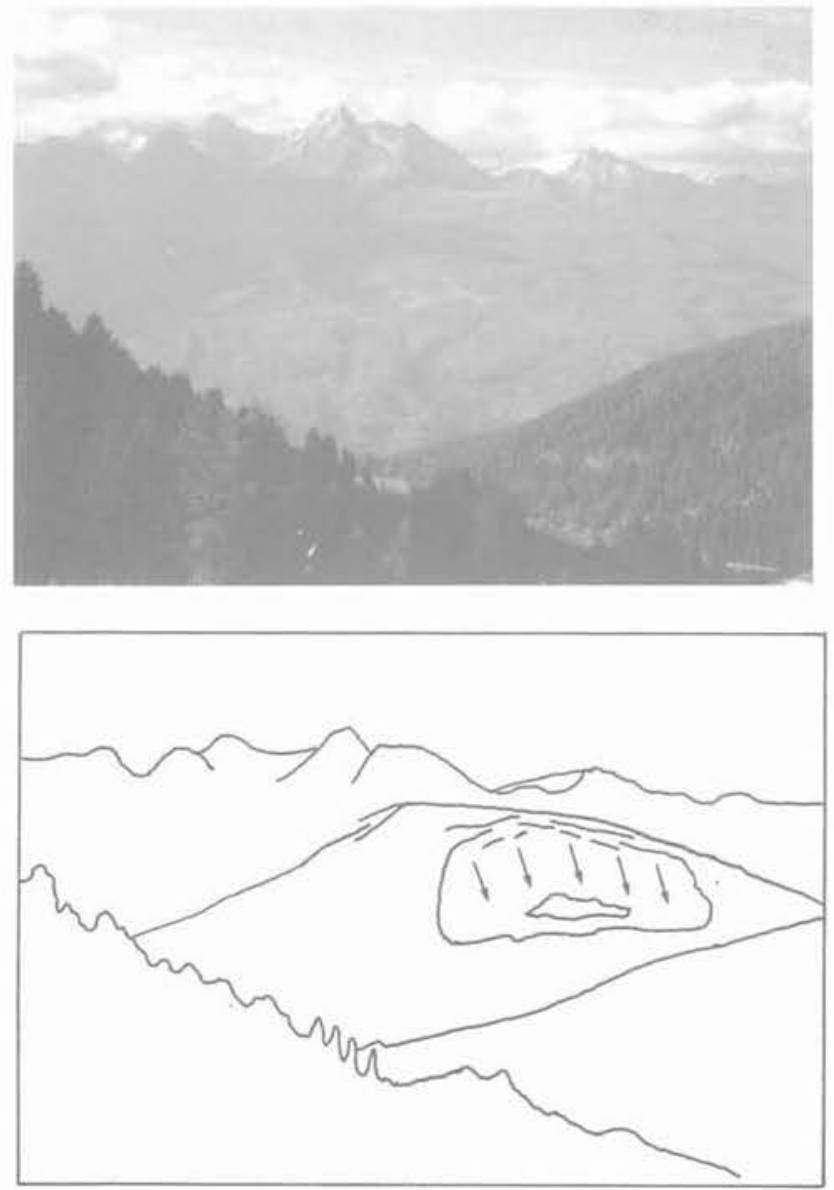

Fig. 2. - Vue générale du glissement au dessus de Valezan.
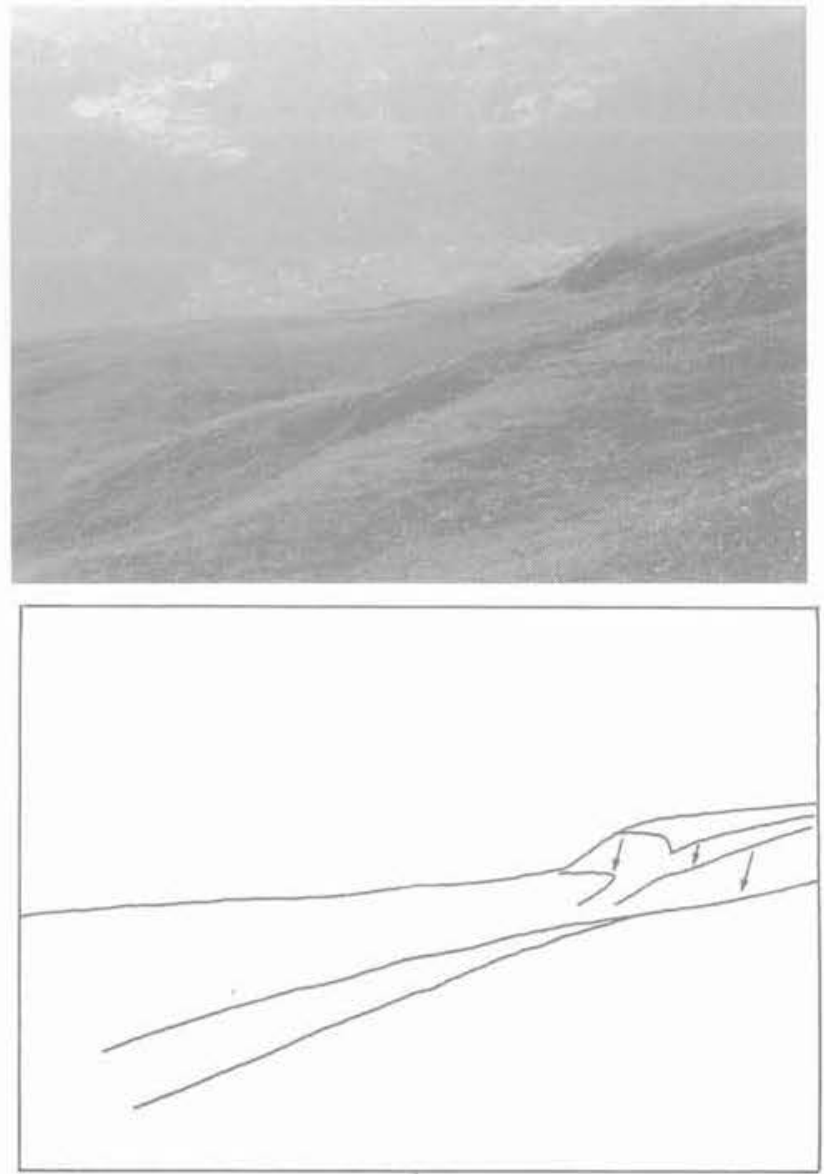

Fig. 3. - Partie sommitale du glissement (photographie en direction d'Aime).
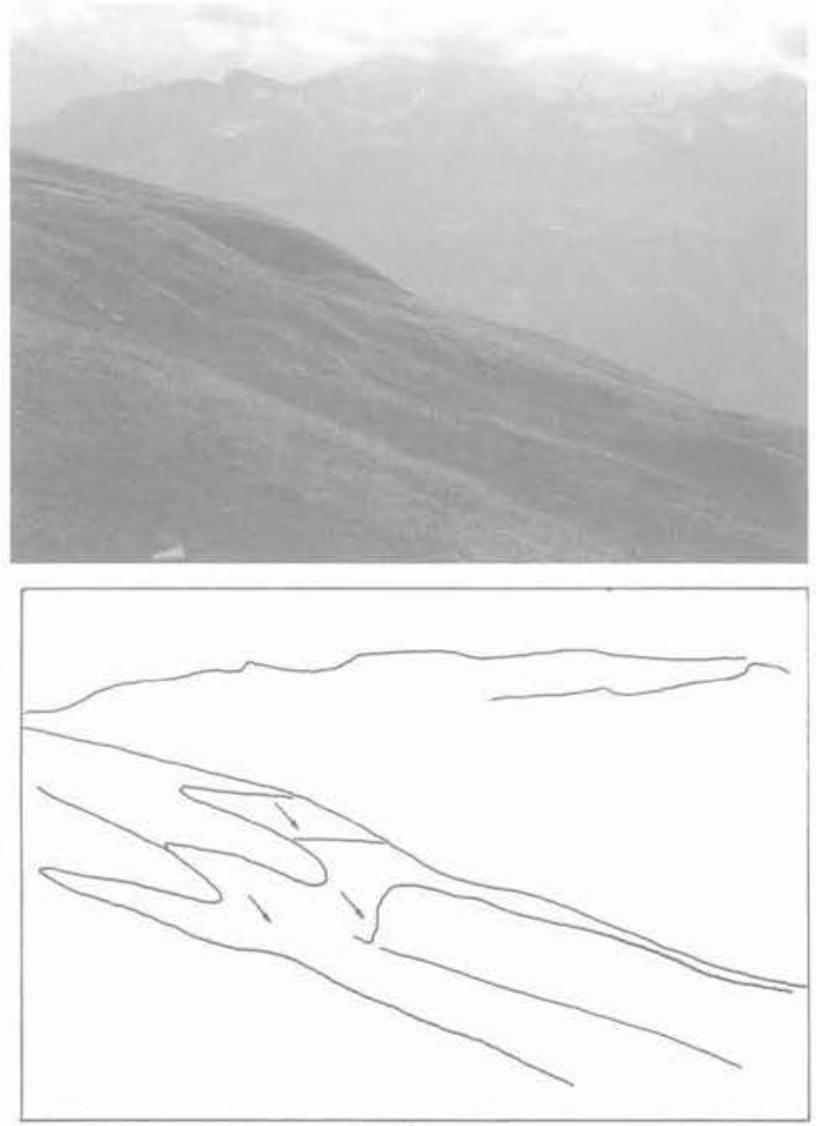

Fig. 4. - Partie sommitale du glissement (photographie en direction de Bourg-St-Maurice). 

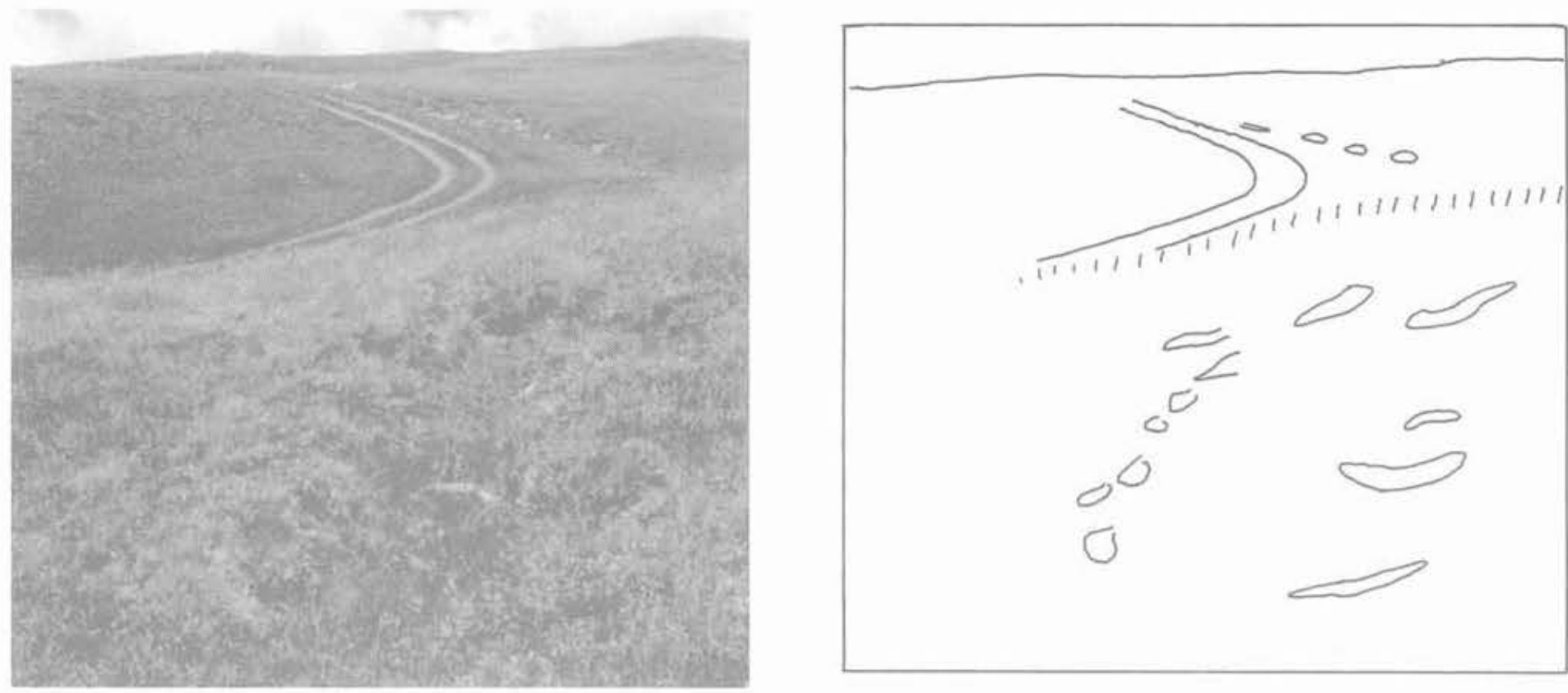

Fig. 5. - Déchirure du tapis végétal par cisaillement montrant l'activité actuelle du glissement sur le flanc droit de la zone en mouvement.

En l'absence d'une reconnaissance géotechnique véritable il apparait que les masses en mouvement ont plus une apparence de sol que de roches. Dans des déblais routiers, dans des petites fouilles, dans des arrachements on observe des terres jaunatres provenant de l'altération de micashistes grisâtres très onctueux au toucher ainsi que leurs produits de décomposition. Il n'y a pas de doute que ces terrains ont un angle de frottement interne faible, qu'ils sont peu perméables et que le rôle de l'eau doit être très important pour l'équilibre général du talus.

Il est évidemment impossible d'évaluer avec précision la masse qui glisse en l'absence de sondages permet- tant de situer la ou les surfaces de glissement. Toutefois, étant donnés les dénivelés des décrochements de tête, la distance qui sépare crêtes et bourrelets successifs en tête et l'ampleur du mouvement, il est difficile de croire que la zone qui glisse a moins de 20 ou même $30 \mathrm{~m}$ d'épaisseur. En surface on identifie avec certitude, entre $2200 \mathrm{~m}$ et $1400 \mathrm{~m}$, une aire de $5 \mathrm{~km}^{2}$ touchée par l'accident, ce qui représente. un volume de 150 millions de mètres cubes de terre en mouvement et situe ce glissement parmi les très grands mouvements alpins. Le glissement serait évidemment encore plus énorme si le mouvement était en liaison avec le toit du houiller profond situé probablement à plusieurs centaines de mètres en dessous de la surface du sol. 